\title{
Hidden aqueductal stenosis associated to bilateral idiopathic foramina of Monro stenosis mimicking a Chiari I malformation? Case report
}

\author{
Andrea Bartoli • Cristina Diana Ghinda • \\ Ivan Radovanovic • Shahan Momjian
}

Received: 25 February 2012 / Accepted: 20 June 2012 /Published online: 7 September 2012

(C) Springer-Verlag 2012

\begin{abstract}
A 39-year old man came to our outpatient clinic with long history of unspecific symptoms and signs. Cerebral MRI showed herniation of the cerebellar tonsils of more than $1 \mathrm{~cm}$ below the foramen magnum and a triventricular hydrocephalus. A diagnosis of Chiari I malformation was retained. After an osteo-dural decompression of the posterior fossa, post-operative MRI revealed an aqueductal stenosis with triventricular hydrocephalus. An endoscopic-thirdventriculostomy showed an idiopathic stenosis of the right foramen of Monro. Residual symptoms and persistence of biventricular hydrocephalus justified a ventriculo-peritoneal shunt. Aqueductal and foramina of Monro stenosis can mimick a Chiari I malformation.
\end{abstract}

Keywords Aqueductal stenosis · Foramen of Monro stenosis · Chiari I malformation · Hydrocephalus

\section{Introduction}

Hydrocephalus was classified by Dandy in 1920 as communicating or obstructive depending on the flow

\footnotetext{
A. Bartoli $\cdot$ I. Radovanovic $\cdot$ S. Momjian

Departement of Neurosurgery, Faculty of Medicine, Geneva University Hospitals,

Geneva, Switzerland

C. D. Ghinda

Faculty of Medicine, University of Montreal,

Montreal, Canada

\author{
A. Bartoli $(\bowtie)$ \\ Service de Neurochirurgie, Geneva University Hospital, \\ 4, Rue Gabrielle Perret-Gentil, \\ 1211 Geneva14, Switzerland \\ e-mail: b_and@hotmail.com
}

of cerebrospinal fluid (CSF) from the ventricles to the subarachnoidal spaces [6].

Amongst the different causes of obstructive (non-communicating) hydrocephalus, aqueductal stenosis (AS) is the most common cause in the adult [19]. AS can be primary (idiopathic), where different kinds of stenosis have been described (atresia, forking, septum, gliosis) [2, 3, 10], or secondary to intracranial space-occupying lesions.

On the other hand, stenosis of one or both foramina of Monro (with subsequent non-communicating hydrocephalus) is a rare condition that can be idiopathic [8] or secondary to infections [1, 8] (with subsequent scarring), vascular malformations, thalamic or intraventricular tumours $[15,21]$.

It is well known that Chiari malformation (CM) is a heterogeneous nosologic entity for which four classic varieties have been described. Except for the fourth one (cerebellar hypoplasia or aplasia), the common feature of the remaining three forms is an abnormal position and/or shape of the cerebellar tonsils herniating to some extent below the foramen magnum with or without hydrocephalus, brainstem deformation, syringomielia, medullary compression and ischemia. Each form has its own clinical and anatomical findings and most cases are congenital, even though few cases of acquired CM I have been reported, such as after lumbar drainage [7, 14, 18].

Natural history, diagnosis and treatment of CM I remain controversial and debatable [13-20].

We present a case of a non-communicating hydrocephalus due to AS associated it to a bilateral idiopathic stenosis of the foramina of Monro mimicking the clinical and radiological findings of a Chiari I malformation.

Idiopathic bilateral stenosis of the foramina of Monro is extremely rare; we found only six cases in the adult population. To our knowledge this is a unique case of bilateral stenosis of foramina of Monro associated to a primary AS in 


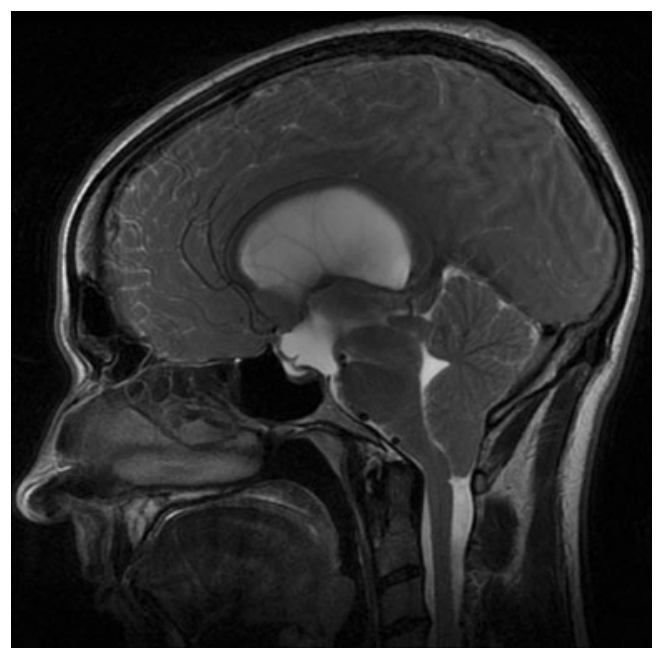

Fig. 1 Preoperative MRI showing a tonsillar herniation below the foramen magnum (17 $\mathrm{mm}$ from the basion) and an obliteration of the cerebellar subarachnoidal spaces

which the clinical and radiological findings led us to consider (and treat) the patient as if he were harbouring a CM I.

\section{Case report}

A 39-year-old right-handed man in good health condition presented to our outpatient clinic reporting daily headaches, mostly positional and increasing with Valsalva's manoeuvre, associated to neck pain. He also complained of some unspecific neurologic symptoms such as memory impairment, occasional visual impairment with scotomas and diplopia, a tinnitus on the right side, paresthesias in the four extremities and in the perioral region.

Clinical examination revealed an intention tremor, a lateral nystagmus on both sides, a slight dysmetria and a positive Romberg's test which worsened after closing the eyes.

A cerebral MRI showed a tonsillar herniation of more than $2 \mathrm{~cm}$ below the foramen magnum (FM) from an apparently relatively small posterior fossa and a bulbar compression against the clivus (Fig. 1). We found a triventricular hydrocephaly with an unusual shape of the third ventricle, with the sole dilation in its anterior part whilst the posterior part appeared normal (Fig. 2). The fourth ventricle was normal in shape and size and there was no syringomielia on the spinal MRI.

This appearance led us to make the diagnosis of a Chiari I malformation causing an obstructive hydrocephalus and to assign the patient's symptoms and signs to a chronic intracranial hypertension.

We therefore performed an osteo-dural decompression of the posterior fossa with a temporary right external occipital shunt. However the post-operative MRI showed welldefined subarachnoidal spaces in the posterior fossa with an AS and the persistence of triventricular hydrocephalus (Fig. 3) as well as a suspicion of bilateral stenosis of the foramina of Monro. The patient described no clear improvement of symptoms. The position of the tonsils now appeared normal as judged in relation to the anterior margin of the FM (basion).

The previously "hidden" AS was the main feature on the post-op MRI, thus explaining the clinical and radiological findings. We decided to treat it by an ETV. Endoscopy confirmed the stenosis of the foramen of Monro which was dilated and passed with the endoscope, and a good fenestration between the floor of the third ventricle and the subarachnoidal space was performed (Fig. 4). Due to the bilateral stenosis of the foramina of Monro, we left a subgaleal reservoir in communication with the right frontal horn in case of obstruction of the fenestration. After this operation the patient described a slight improvement of his symptoms but he was still suffering from headache and nausea. His symptoms improved after tapping $40 \mathrm{cc}$ of CSF. The CT scan still showed a biventricular hydrocephalus with a normal third ventricle (Fig. 5). Because of these residual symptoms we placed a ventriculo-peritoneal shunt that was eventually changed, once, after a shunt infection.
Fig. 2 Preoperative MRI showing a triventricular hydrocephalus with an unusual shape of the third ventricle, squeezed posteriorly (a) and dilated anteriorly (b). Note the swelling of the optic sheaths on the left side (c)

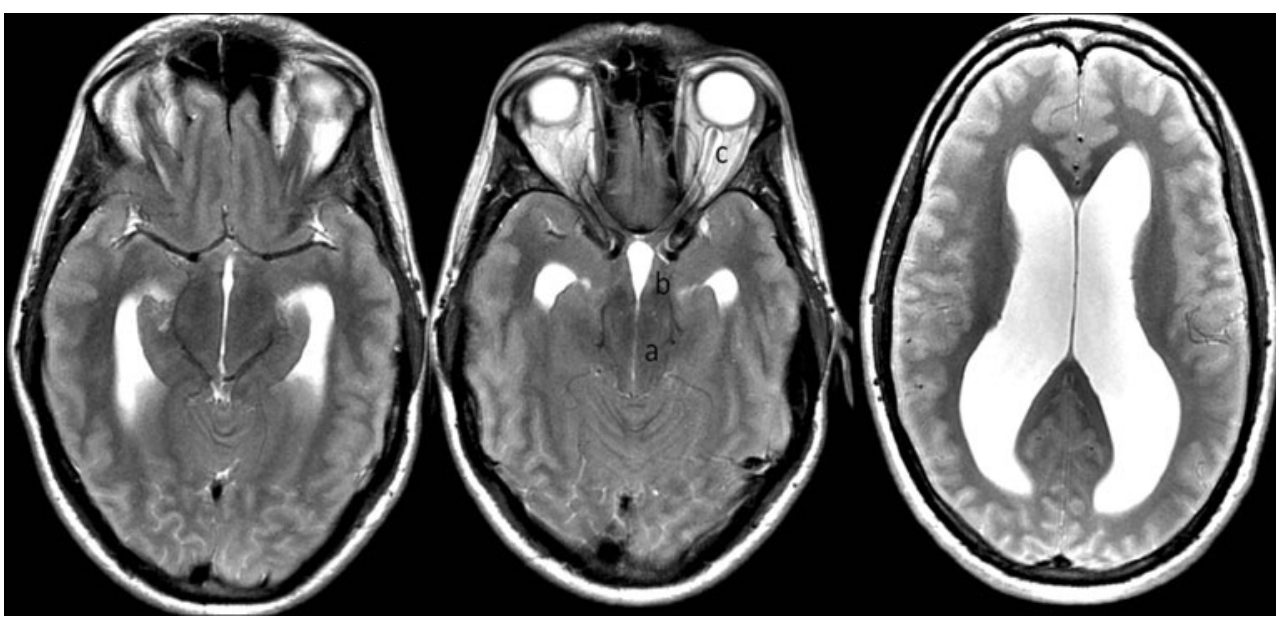




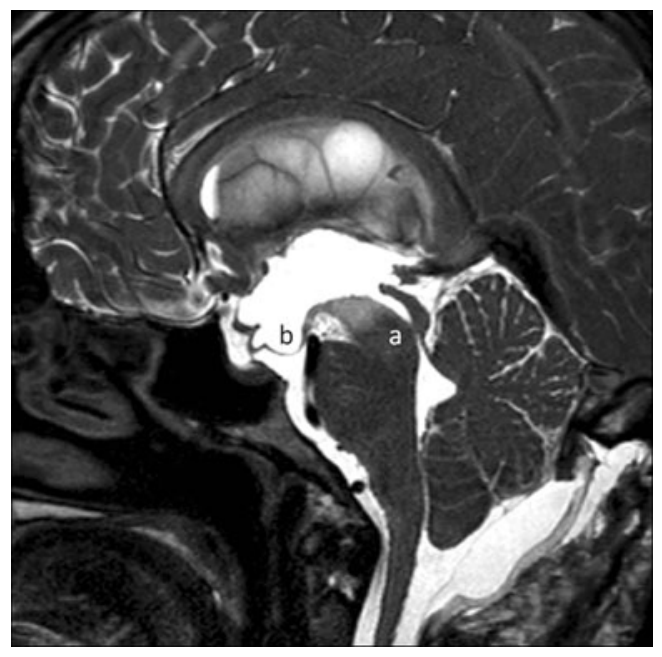

Fig. 3 First post operative MRI: after osteo-dural decompression cerebellar CSF spaces are more visible and an aqueductal stenosis is revealed (a). Typical bulging of the floor of the third ventricle is also present (b)

\section{Discussion}

The most common cause of noncommunicating hydrocephalus in adults is AS [19], whilst we found only one report of bilateral idiopathic stenosis of the foramina of Monro [8]. To our knowledge there are no reports of the association of AS and bilateral stenosis of interventricular foramina. These conditions cause a ventricular dilation and may lead to a chronic intracranial hypertension, eventually pushing the intracranial content downward through the foramen magnum.

On the other hand, CM I has neither specific symptoms nor signs, but it tends to be rather considered as a clinical syndrome. As reported by Milhorat et al. in their series of 364 patients, the clinical syndrome variably associates headaches with visual, otoneurological, lower cranial nerves, brainstem, cerebellar, spinal cord and nonspecific disturbances [12].

Clinically our patient presented at least one symptom or sign of each of those disturbances. Tremor has also been reported as the sole symptom/sign of a hydrocephalus due to an AS [9].
In our patient we faced a diagnostic and therapeutic dilemma, particularly from a radiological point of view, as the clinical presentation was quite unspecific.

The most-used radiological diagnostic criterion on MRI for CM I is the downward herniation of cerebellar tonsils (at least $5 \mathrm{~mm}$ ) [12], obliteration of the retrocerebellar cerebrospinal fluid spaces and a varying degree of cranial base dysplasia (such as shortening of the length of the clivus and/or length of the supraocciput line from opisthion to internal occipital protuberance, increased angle between tentorium and the supraocciput line) [12]. Furthermore, volumetric calculations of posterior fossa and cerebrospinal fluid volume are also available as a diagnostic tool $[5,11$, 17], and usually reveal a volume reduction [4].

In our case the first cerebral MRI showed herniation of cerebellar tonsils $17 \mathrm{~mm}$ below the FM with an obstructive hydrocephalus involving mainly lateral ventricles and, with an unusual configuration, the third ventricle, plus an obliteration of the retrocerebellar cerebrospinal fluid spaces. Compared with cranial base linear measurements done by Milhorat et al. in their series [12], we found a reduction of the length of the clivus $(33,3 \mathrm{~mm})$ and of the supraocciput line $(39,8 \mathrm{~mm})$ and a certain kinking of the brain stem against the clivus.

However, volumetric calculation of the posterior fossa using the Cavalieri's estimator $[5,11,16]$ showed a volume of $160.68 \mathrm{ml}$, which more closely matches those values belonging to control subjects in the Milhorat series [12].

Considering the unspecific clinical findings combined with those radiological findings, we diagnosed a CM I malformation that motivated an osteodural decompression of the posterior fossa and an external shunt in the right occipital horn.

The first post-operative course was uneventful but no real clinical improvement occurred. The first post-operative cerebral MRI showed an enlargement of the cerebellar subarachnoid spaces, an ascent of the cerebellar tonsils but, above all, an AS (Fig. 3) that was not visible on the initial MRI. The shape of the third ventricle (dilated only anteriorly and squeezed in its posterior part) suggested an association of a
Fig. 4 Endoscopic view of the lateral right ventricle with a barely visible foramen of Monro (a). MRI after EVT shows a good patency through the floor of the third ventricle (b). Cerebellar tonsils are $5 \mathrm{~mm}$ below the basion

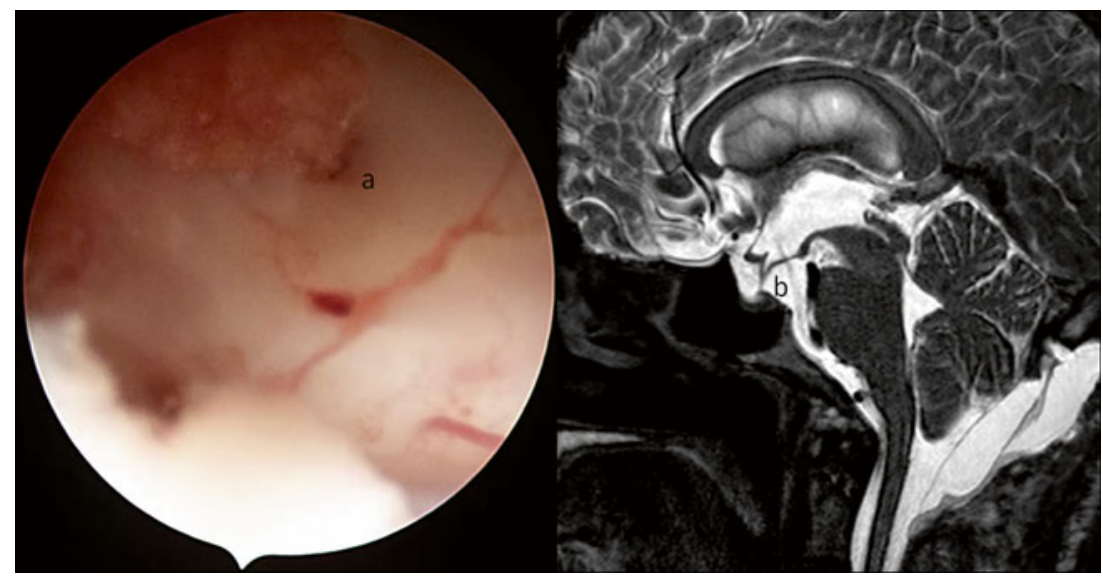




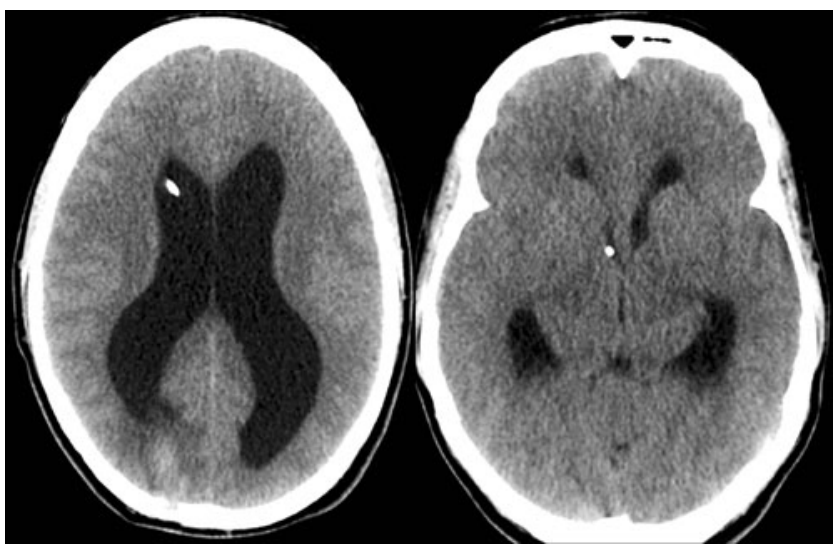

Fig. 5 Cerebral CT scan after EVT: the third ventricle dilation resolved whilst a biventricular hydrocephalus is still present, reflecting the bilateral stenosis of the foramina of Monro. A subgaleal reservoir is present

bilateral stenosis of both foramena of Monro (poorly visible on our MRI but confirmed endoscopically), thus explaining the dilation of lateral ventricles.

From a pathophysiological point of view we hypothesize that a high pressure gradient ought to exist between the lateral ventricles and the third ventricle as the only reasonable explanation of a such a distortion of the third ventricle (Fig. 2). No studies have been published on the pressure gradient inside the ventricular system in obstructive hydrocephalus, but it is admitted that a very low gradient should exist in communicating hydrocephalus between the ventricular system and the subarachnoid spaces [9].

In this case the ODD "revealed" an AS and a bilateral stenosis of a foramen of Monro: in our opinion both conditions should have been treated by a septostomy with a dilatation of a foramen of Monro and an EVT or, alternatively, by a septostomy with a dilatation of a foramen followed by a shunt. Indeed, as suggested retrospectively by the volumetric estimation of the posterior fossa that matched control values, the diagnosis was this association of obstructive hydrocephalus rather than CM I and the ODD therefore appeared unnecessary. Furthermore, even if the diagnosis of CM I is retained, it has been advocated by different authors that the treatment of hydrocephalus in CM I should precede the ODD, as it can improve the overcrowding of the posterior fossa contents, thereby correcting the CM I, and also the syringomyelia [22].

\section{Conclusion}

This is an interesting case showing an uncommon association of an AS and a stenosis of both foramina of Monro with some radiological features of a CM I.

Diagnosis of CM I can be very tricky and sometimes remains unclear. CM I can be mimicked radiologically and clinically by this association of ventricular obstructions.
Conflicts of interest None

\section{References}

1. Alexander E Jr, Bottarell EH (1949) Unilateral hydrocephalus resulting from occlusion of foramen of Monro. Complication of radical removal of brain abscess. J Neurosurg 6:197-206

2. Arienta C, Capricci E, Baiguini M (1986) Non tumoral aqueduct stenosis. Multiple microscopic subependymal glial protuberances demonstrated at histological examination. J Neurosurg Sci 30:67-70

3. Balestrazzi P, de Gressi S, Donadio A, Lenzini S (1989) Periaqueductal gliosis causing hydrocephalus in a patient with neurofibromatosis type 1 . Neurofibromatosis 2:322-325

4. Caldarelli M, Novegno F, Di Rocco C (2009) A late complication of CSF shunting: acquired Chiari I malformation. Childs Nerv Syst 25(4):443-445

5. Clatterbuck RE, Sipos EP (1997) The efficient calculation of neurosurgically relevant volumes from computed tomographic scans using Cavalieri's direct estimator. Neurosurgery 40:339-343

6. Dandy W (1920) The diagnosis and treatment of hydrocephalus resulting from strictures of the aqueduct of Sylvius. Surg Gynaecol Obstet 31:340-358

7. Huang PP, Constantini S (1994) "Acquired Chiari I malformation". Case report (1994). J Neurosurg 80:1099-1102

8. Kalhorn SP, Strom RG, Harter DH (2011) Idiopathic bilateral stenosis of the foramina of Monro treated using endoscopic foraminoplasty and septostomy. Neurosurg Focus 30(4):E5

9. Levine DN (2008) Intracranial pressure and ventricular expansion in hydrocephalus: have we been asking the wrong question? J Neurol Sci 269:1-11

10. Mangano FT, McAllister JP, Jones HC, Johnson MJ, Kriebel RM (1998) The microglial response to progressive hydrocephalus in a model of inherited aqueductal stenosis. Neurol Res 20:697-704

11. Mayhew TM, Olsen DR (1991) Magnetic resonance imaging (MRI) and model free estimates of brain volume determined using the Cavalieri principle. J Anat 178:133-144

12. Milhorat TH, Chou MW, Trinidad EM, Kula RW, Mandell M, Wolpert C, Speer MC (1999) Chiari I malformation redefined: clinical and radiographic findings for 364 symptomatic patients. Neurosurg 44:1005-1017

13. Novegno F, Caldarelli M, Massa A, Chieffo D, Massimi L, Pettorini B, Tamburrini G, Di Rocco C (2008) The natural history of the Chiari type I anomaly. J Neurosurg Pediatr 2:179-187

14. Payner TD, Prenger E, Berger TS, Crone KR (1994) Acquired Chiari malformations: incidence, diagnosis, and management. Neurosurg 34(3):429-434

15. Pfeiffer G, Friede RL (1984) Unilateral hydrocephalus from early developmental occlusion of one foramen of Monro. Acta Neuropathol 64:75-77

16. Rose GD, Harry JD (1990) Brain volume estimation from serial section measurements: a comparison of methodologies. J Neurosci Methods 35:115-124

17. Seiler FA, Lew SM (2010) Aqueductal stenosis presenting as isolated tremor: case report and review of the literature. Ped Neurosurg 46:392-395

18. Sathi S, Stieg PE (1993) "Acquired" Chiari I malformation after multiple lumbar punctures: case report. Neurosurg 32:306-309

19. Tisell M (2005) How should primary aqueductal stenosis in adults be treated? - A review. Acta Neurol Scand 111:145-153

20. Tubbs RS, Lyerly MJ, Loukas M, Shoja MM, Oakes WJ (2007) The pediatric Chiari I malformation: a review. Childs Nerv Syst 23:1239-1250 
21. Wilberger JE Jr, Vertosick FT Jr, Vries JK (1983) Unilateral hydrocephalus secondary to congenital atresia of the foramen of Monro. J Neurosurg 59(5):899-901

22. Massimi L, Pravatà E, Tamburrini G, Gaudino S, Pettorini B, Novegno F, Colosimo C Jr, Di Rocco C (2011) Endoscopic third ventriculostomy for the management of Chiari I and related hydrocephalus: outcome and pathogenetic implications. Neurosurg 68(4):950-956

\section{Comment}

In this paper, the authors report an intriguing case of a Chiari malformation type I associated with a stenosis of bilateral foramina Monroi, which was misdiagnosed before surgery. The authors suppose that a high pres- sure gradient between the lateral ventricles and the third ventricle is the cause of the deformity of the third ventricle. In fact, to my knowledge, no enquiries have been published on the pressure gradient inside the ventricular system in obstructive hydrocephalus. The case is attractive and may support our observations of other cases of Chiari malformation with postoperative CSF flow dysregulation. This can be reasonably explained by a hidden obstructive hydrocephalus and confirms the hypothesis, that the Chiari malformation should be assimilated to a CSF flow impairment. Research studies should be performed in order to gain more insight into the pathophysiology of these pathologies.

Alex Alfieri

Sebastian Simmermacher

Gershom Koman

Halle (Saale), Germany 